\title{
Produção de cerâmica vermelha utilizando argila da mineração de gipsita e resíduo de gesso
}

\author{
Pâmela Bento Cipriano ${ }^{a b^{*}}$, Rafaela Tatianne Oliveira de Rezendea, Andréa de Vasconcelos \\ Ferrazab $^{b}$ \\ a Grupo de Pesquisa em Materiais, Universidade Federal do Vale do São Francisco, Juazeiro, 48902-300, Bahia, Brasil. \\ b Pós-graduação em Ciências dos Materiais, Universidade Federal do Vale do São Francisco, Juazeiro, 48902-300, Bahia, Brasil. \\ *pamela.ufcg@gmail.com
}

Recebido: 14 outubro 2018 / Aceito: 1 dezembro 2018 / Publicado online: 2 janeiro 2019

\begin{abstract}
Resumo
Os resíduos de gesso e de argila são gerados ao longo da cadeia produtiva do gesso. O objetivo dessa pesquisa foi avaliar os efeitos da incorporação de resíduo de gesso da indústria de placas em argila residual obtida da extração da gipsita, para obtenção de produtos de cerâmica vermelha com melhores propriedades reduzindo os impactos ambientais. Foi avaliada a incorporação de 5\% e 10\% de resíduos de gesso na argila, considerando duas temperaturas de sinterização $\left(800\right.$ e $\left.1050{ }^{\circ} \mathrm{C}\right)$, sendo determinada a retração linear, a absorção de água, a porosidade e a massa específica aparente, a perda ao fogo e a resistência à flexão. Avaliou-se a morfologia da superfície de fratura por microscopia. A resistência à flexão foi cerca de 4,24\% e a absorção de água aproximadamente 11,45\%. A formação de pequenos aglomerados e presença de poros foram evidenciadas pelas microscopias nas amostras contendo gesso independente do teor e das temperaturas de queima estudadas. Melhores resultados foram obtidos com a adição de $5 \%$ de resíduo de gesso queimadas a $800{ }^{\circ} \mathrm{C}$ apresentando propriedades semelhantes à argila pura. A utilização de materiais residuais permite a redução dos impactos ambientais e a possibilidade de novas formulações para produtos de cerâmica vermelha.
\end{abstract}

Palavras-chave: Reaproveitamento, produto cerâmico, impacto ambiental.

\section{Production of red ceramics using gypsum mining clay and gypsum waste}

\begin{abstract}
Waste of gypsum and clay are generated along the productive chain of gypsum. The objective of this research was to evaluate the effects of the incorporation of gypsum waste from the slab industry into residual clay obtained from gypsum extraction to obtain red ceramic products with better properties reducing environmental impacts. The incorporation of 5\% and 10\% of gypsum waste in the clay and considering two sintering temperatures $\left(800\right.$ and $\left.1050{ }^{\circ} \mathrm{C}\right)$ was evaluated. Linear retraction, water absorption, porosity and apparent specific mass, fire loss and flexural strength were determined. The morphology of the fracture surface was evaluated by microscopy. The flexural strength was about $4.24 \%$ and the water absorption was about $11.45 \%$. The formation of small agglomerates and presence of pores were evidenced by microscopy in samples containing gypsum independent of the content and the burning temperatures studied. Better results were obtained with the addition of 5\% of gypsum residue burned at $800{ }^{\circ} \mathrm{C}$ presenting properties similar to pure clay. The use of waste materials allows the reduction of environmental impacts and the possibility of new formulations for red ceramic products.
\end{abstract}

Keywords: Reuse, ceramic product, environmental impact.

\section{Introdução}

Atualmente, vêm crescendo a pesquisa e o desenvolvimento de ações que envolvam a coleta e a reciclagem dos resíduos com o foco na minimização dos impactos ao meio ambiente ocasionados pelo alto volume de resíduos sólidos gerados pelas indústrias (Morini, Oliveira, Pereira \& Hotza, 2016; Cabral \& Azevedo, 2017).

$\mathrm{Na}$ mineração existe uma grande preocupação com a disposição dos resíduos produzidos ao longo do processo produtivo. No Brasil, no estado de Pernambuco possui reservas abundantes de gipsita na região do Sertão do Araripe (Barbosa, Ferraz \& Santos, 2014). Segundo Araújo (2004) quando a gipsita é extraída, o solo removido apresenta grande concentração de argila que pode ser utilizada como matéria prima para a fabricação de tijolos, telhas e outros produtos. $\mathrm{O}$ armazenamento inadequado destes materiais provoca $\mathrm{o}$ 
aumento do volume de sedimentos nos cursos d'água existentes na região e impactam a ecologia local.

$O$ resíduo de gesso pode ser gerado nas indústrias e na construção civil, e quando descartado de forma inapropriada, pode se solubilizar no solo e contaminar os lençóis freáticos, pois na presença de umidade e de microrganismos, provoca a dissociação dos componentes do resíduo em dióxido de carbono, água e gás sulfídrico (Carvalho, Gomes, Pinheiro, Lima \& Silva, 2015).

Para viabilizar a redução dos efeitos negativos referentes aos problemas ambientais é importante explorar as matérias primas e as possibilidades de novas composições (Moreno, Bartolomeu \& Lima, 2009). A incorporação de resíduos nos processos cerâmicos representa uma alternativa a sua reciclagem, economia de matérias primas, redução dos custos e contribui com a sustentabilidade (Menezes et al., 2007).

Estudos têm sido realizados buscando avaliar as propriedades de produtos cerâmicos com a adição de resíduo de gesso, principalmente para beneficiar a construção civil (Sousa, 2014).

O objetivo dessa pesquisa foi avaliar os efeitos da incorporação de resíduo de gesso da indústria de placas em argila residual obtida da extração da gipsita, para obtenção de produtos de cerâmica vermelha com melhores propriedades reduzindo os impactos ambientais.

\section{Material e Métodos}

As matérias primas utilizadas para a realização deste estudo foram: resíduos de gesso $(\mathrm{RG})$ e argila $(\mathrm{C})$. O resíduo de gesso foi coletado em uma empresa de produção de placas (Juazeiro, Bahia). A argila residual proveniente do capeamento da gipsita foi fornecida pela empresa Ecogesso (Araripina, Pernambuco).

Para cada matéria prima, foi determinada a distribuição granulométrica, utilizando as peneiras $n^{\circ} 40, n^{\circ} 50, n^{\circ} 80$ e $n^{\circ}$ 200 (Associação Brasileira de Normas Técnicas [ABNT], 2005) em série, em agitador mecânico (Solotest) por $15 \mathrm{~min}$. Para a preparação dos corpos de prova cerâmicos, a argila e o resíduo de gesso foram peneirados, em peneiras $A B N T n^{\circ} 80$ e 100 , respectivamente.

Foram misturados $10 \%$ de água e resíduo de gesso $(5 \%$ e $10 \%$ ) na massa cerâmica. Após $24 \mathrm{~h}$ foram prensadas 5 amostras para cada composição utilizando um molde de dimensão $60 \times 20 \times 5 \mathrm{~mm}^{3}$ em prensa hidráulica (Bovenau P30 ST), aplicando uma carga de 2 toneladas durante $15 \mathrm{~s}$, seguida de um aumento de carga para 6 toneladas por $30 \mathrm{~s}$. As amostras foram secas a $110^{\circ} \mathrm{C}$ durante $24 \mathrm{~h}$ e sinterizadas na mufla a 800 e $1050{ }^{\circ} \mathrm{C}$ durante $2 \mathrm{~h}$. Em seguida, determinaram-se as propriedades: retração linear de queima, a absorção de água, a porosidade e a massa específica aparente, a perda ao fogo e a resistência à flexão, todos conforme metodologia para ensaios preliminares de argilas proposta por Santos et al. (1992, Tabela 1).

Assim, o planejamento experimental foi constituído pela argila pura ( $0 \%$ de resíduo de gesso) e por duas concentrações de resíduo de gesso (5\% e $10 \%$ ) submetidas a duas diferentes temperaturas de sinterização $\left(800\right.$ e $\left.1050{ }^{\circ} \mathrm{C}\right)$. Cada tratamento utilizou-se 5 corpos de prova.
Os corpos de prova foram submetidos ao ensaio de flexão de três pontos, utilizando uma velocidade de ensaio de $0,5 \mathrm{~mm} \cdot \mathrm{min}^{-1}$, em um equipamento universal de ensaios mecânicos (EMIC-DL 1000). Os corpos de prova foram apoiados no suporte a $1 \mathrm{~cm}$ em cada extremidade e a punção centralizado ao meio.

Tabela 1. Equações utilizadas para a determinação dos parâmetros avaliados nos corpos de provas experimentais (Santos, 1992).

\begin{tabular}{l|c}
\hline \multicolumn{1}{c|}{ Parâmetro } & Equação \\
\hline Retração linear de queima (Rl) & $R_{l}=\left(\frac{L_{i}-L_{f}}{L_{i}}\right) \times 100$ \\
\hline Absorção de água (AA) & $A A=\frac{m_{u}-m_{s}}{m_{s}} \times 100$ \\
\hline Massa específica aparente (MEA) & $\mathrm{MEA}=\frac{M s}{M u-M i} \rho_{L}$ \\
\hline Porosidade aparente (PA) & $\mathrm{PA}=\frac{(M u-M q)}{(M u-M i)} \times 100$ \\
\hline Perda ao fogo (PF) & $\mathrm{PF}=\frac{(M s-M q)}{\mathrm{Mq}} \times 100$ \\
\hline Resistência à flexão $(\sigma)$ & $\sigma=\frac{3 \mathrm{Fl}}{2 \mathrm{~b} \mathrm{~d} d^{2}}$ \\
\hline
\end{tabular}

Legenda: ms: massa seca; mu: massa úmida (Mu); mi: massa imersa; mq: massa queimada; F: força máxima na fratura; 1 : distância entre os apoios; b: largura; d: espessura do corpo de prova.

A morfologia da superfície de fratura foi avaliada por microscopia ótica (Marte) com aumento de 20x e por um microscópio eletrônico de varredura (Tescan, modelo Vega $3 \mathrm{XMU})$ com aumento de 4000x. As amostras foram recobertas com ouro durante $10 \mathrm{~min}$ e corrente de 15mA·5min-1 (Quorum, Q150R ES).

Os resultados obtidos foram submetidos à análise de variância (ANOVA). $\mathrm{O}$ teste de $\mathrm{F}$ foi considerado significativo para valores menores que $5 \%$ de probabilidade, logo se executou o de teste de Tukey para 5\% de probabilidade na comparação das médias com o auxílio do programa $\mathrm{R}$.

\section{Resultados e Discussão}

A caracterização granulométrica das matérias primas analisada está descrita na Tabela 2. Logo, a argila apresentou maior quantidade de material fino em relação ao resíduo de gesso. Na fabricação de produtos de cerâmica vermelha a granulometria pode influenciar o empacotamento das partículas durante a prensagem e nas reações que ocorrem na etapa de sinterização devido à área de contato (Moreno et al., 2009; Santos et al., 2017).

Tabela 2. Fração acumulada passante em função do tamanho das partículas obtidas no ensaio de composição granulométrica por peneiras para a argila e o resíduo de gesso.

\begin{tabular}{ccc} 
Tamanho de & \multicolumn{2}{c}{ Fração passante $(\%)$} \\
\cline { 2 - 3 } partícula $(\mu \mathrm{m})$ & Argila & Resíduo de gesso \\
\hline 425 & 37,81 & 19,56 \\
300 & 31,30 & 8,67 \\
180 & 20,01 & 1,41 \\
75 & 7,42 & 0,08 \\
\hline
\end{tabular}

Todos os experimentos apresentaram um aumento na retração linear com o incremento da temperatura (Figura 1). Este comportamento corresponde a melhor sinterização das 
cerâmicas em temperaturas mais altas (Urbánek, Hamácek, Machácek, Kutzendörfer \& Hubálková, 2017). Na temperatura de $800{ }^{\circ} \mathrm{C}$, a retração linear não foi influenciada de forma significativa com a adição de gesso.
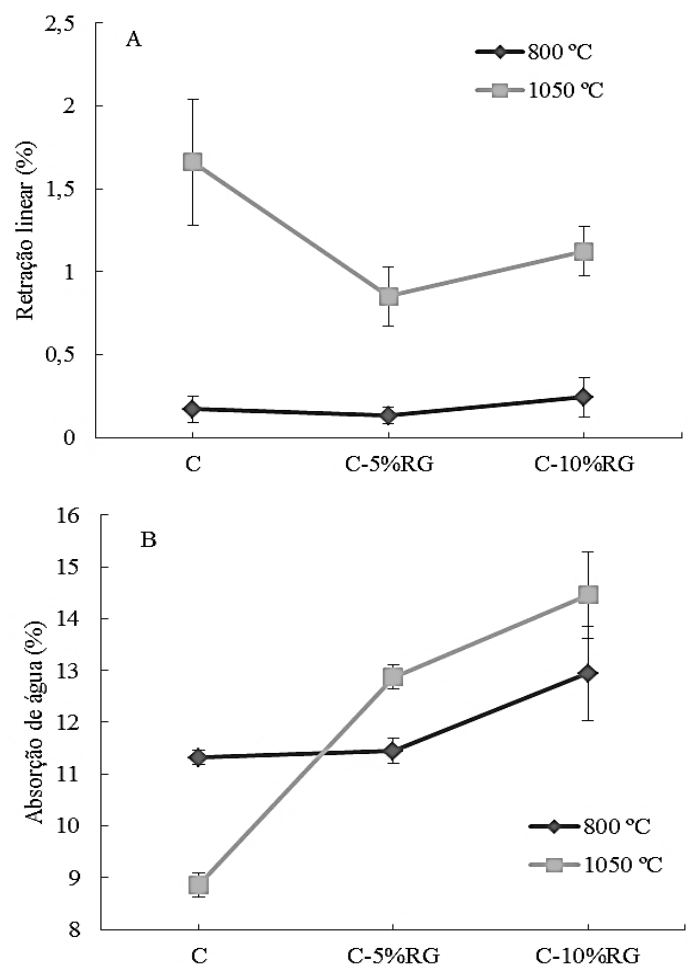

Figura 1. Retração linear de queima (A) e Absorção de água (B) dos corpos de prova cerâmicos de argila (C), contendo $5 \%$ (C-5\%RG) e 10\% (C-10\%RG) de resíduo de gesso, sinterizados nas temperaturas de 800 e $1050{ }^{\circ} \mathrm{C}$.

As A absorção de água não apresentou variação significativa nos experimentos a $800{ }^{\circ} \mathrm{C}$. A argila pura reduziu $2,46 \%$ na absorção de água quando queimada a $1050{ }^{\circ} \mathrm{C}$. A porosidade em um material cerâmico tende a ser menor à medida que a temperatura de queima aumenta devido à densificação que ocorre durante a sinterização fechando os poros, diminuindo a porosidade (Dutra, Silva \& Varela, 2008), isso foi observado nesta pesquisa (Tabela 3).

Nos corpos de prova contendo resíduo de gesso, houve maior absorção de água e a porosidade aparente proporcional ao teor de gesso incorporado e a temperatura de queima. $\mathrm{O}$ aumento da porosidade com a incorporação de gesso a 1050 ${ }^{\circ} \mathrm{C}$ pode ser associado à liberação de gás devido à decomposição parcial do sulfato de cálcio (Silva, 2008).

Todas as composições experimentais atenderam as especificações para a fabricação de blocos cerâmicos para alvenaria de vedação segundo a norma NBR 1520-1 (2005), pois a absorção de água foi entre 8 e $22 \%$.

A incorporação de gesso reduziu a propriedade mecânica independente do teor utilizado quando comparado com a argila sem resíduo, no entanto, a $1050{ }^{\circ} \mathrm{C}$, essa diminuição foi mais significativa com a adição de $10 \%$ de resíduo de gesso em relação aos corpos de prova de argila pura.

As propriedades mecânicas podem ser reduzidas pela presença de poros e aglomerados que atuam como concentradores de tensão (Figuras 2 e 3). Esse resultado está coerente com a maior absorção de água e porosidade aparente nas composições experimentais sinterizadas a $1050{ }^{\circ} \mathrm{C}$. Resultados reportados na literatura mostram uma tendência na redução da resistência à flexão com o aumento no teor de resíduo de gesso e da temperatura de sinterização, logo foram obtidos valores semelhantes aos encontrados nesse trabalho para a resistência a flexão de $2,5 \mathrm{MPa}$ para corpos cerâmicos com $10 \%$ de resíduo de gesso queimados a $1050{ }^{\circ} \mathrm{C}$ (Silva, 2018).

Os corpos de prova sinterizados a $800^{\circ} \mathrm{C}$ não apresentaram variação significativa para a perda ao fogo e a massa específica aparente em todas as composições experimentais analisadas (Tabela 4). Em contrapartida, quando sinterizadas à $1050^{\circ} \mathrm{C}$, observou-se redução de massa específica já no uso de $5 \%$ de resíduo de gesso. Houve aumento de perda ao fogo em ambas as temperaturas de sinterização, sendo a maior perda nos corpos de prova com a adição de $10 \%$ de gesso, submetidos a temperatura de $1050{ }^{\circ} \mathrm{C}$. Essa perda é proporcional ao teor de gesso e está associada a maior porosidade (Tabela 4). Contribuindo com uma redução na massa específica aparente tornando o material mais leve, pois a massa específica aparente está diretamente ligada à porosidade aparente das amostras (Santis, Sichieri, Rossignolo, Ferreira \& Fiorelli, 2013).

As amostras apresentaram perda ao fogo entre 8,57 e $13,66 \%$ (Tabela 4). Isso pode estar relacionado à perda de águas coordenadas e adsorvidas, hidroxilas dos argilominerais, carbonatos, matéria orgânica e outros (Pereira et al., 2014).

Tabela 3. Valores percentuais médios para a Retração Linear de Queima (RLQ), Absorção de Água (AA), Porosidade Aparente (PA) e Resistência à Flexão (RF, em MPa) segundo a variação da temperatura $(\mathrm{T})$ e concentração de resíduo de gesso $(\mathrm{CRG})$.

\begin{tabular}{|c|c|c|c|c|c|c|c|c|}
\hline \multirow{3}{*}{ CRG (\%) } & \multicolumn{8}{|c|}{ Temperatura $\left({ }^{\circ} \mathrm{C}\right)$} \\
\hline & \multicolumn{2}{|c|}{ RLQ } & \multicolumn{2}{|c|}{ AA } & \multicolumn{2}{|c|}{ PA } & \multicolumn{2}{|c|}{ RF } \\
\hline & 800 & 1050 & 800 & 1050 & 800 & 1050 & 800 & 1050 \\
\hline 0 & $0,17^{\mathrm{bA}}$ & $1,66^{\mathrm{aA}}$ & $11,32^{\mathrm{aB}}$ & $8,85^{\mathrm{bC}}$ & $20,59^{\mathrm{aB}}$ & $17,80^{\mathrm{bC}}$ & $4,65^{\mathrm{bA}}$ & $7,24^{\mathrm{aA}}$ \\
\hline 5 & $0,10^{\mathrm{bA}}$ & $0,85^{\mathrm{aB}}$ & $11,45^{\mathrm{bB}}$ & $12,78^{\mathrm{aB}}$ & $21,32^{\mathrm{bB}}$ & $24,27^{\mathrm{aB}}$ & $4,24^{\mathrm{aA}}$ & $2,79^{\mathrm{bB}}$ \\
\hline 10 & $0,42^{\mathrm{bA}}$ & $1,12^{\mathrm{aB}}$ & $12,94^{\mathrm{bA}}$ & $14,46^{\mathrm{aA}}$ & $24,04^{\mathrm{bA}}$ & $26,10^{\mathrm{aA}}$ & $4,04^{\mathrm{aA}}$ & $1,25^{\mathrm{bB}}$ \\
\hline
\end{tabular}


Tabela 4. Percentual médio da perda ao fogo (PF) e da massa específica aparente (MEA) segunda o percentual de resíduo de gesso (CRG) sinterizados em diferentes temperaturas.

\begin{tabular}{cccccc}
\hline \multirow{2}{*}{$\begin{array}{c}\text { CRG } \\
(\%)\end{array}$} & \multicolumn{3}{c}{ MEA } & \multicolumn{2}{c}{ PF } \\
\cline { 2 - 3 } \cline { 5 - 6 } & 800 & 1050 & & 800 & 1050 \\
\hline 0 & $1,82 \pm 0,04^{\mathrm{bA}}$ & $2,01 \pm 0,02^{\mathrm{aA}}$ & & $8,57 \pm 0,14^{\mathrm{aB}}$ & $8,71 \pm 0,24^{\mathrm{aC}}$ \\
5 & $1,86 \pm 0,03^{\mathrm{aA}}$ & $1,87 \pm 0,03^{\mathrm{aB}}$ & & $8,60 \pm 0,12^{\mathrm{bB}}$ & $10,73 \pm 0,18^{\mathrm{aB}}$ \\
10 & $1,84 \pm 0,05^{\mathrm{aA}}$ & $1,81 \pm 0,03^{\mathrm{aC}}$ & & $9,36 \pm 0,68^{\mathrm{bC}}$ & $13,66 \pm 0,30^{\mathrm{aA}}$
\end{tabular}

Médias seguidas de mesma letra maiúscula na coluna e minúscula na linha (entre as temperaturas 800 e 1050 de cada variável) não diferem entre si pelo teste de Tukey $(\mathrm{p}<0,05)$.

As micrografias obtidas da superfície de fratura dos corpos de prova experimentais revelaram a presença de rugosidades (Figuras 2 e 3), com má distribuição das partículas de gesso nas amostras que contém esse resíduo, formando pequenos aglomerados que pode estar atuando como defeito, reduzindo as propriedades mecânicas.

Observou-se uma microestrutura heterogênea com poros e aglomerados sugerindo que a sinterização foi parcial nas amostras a $1050^{\circ} \mathrm{C}$ (Figura 3). Resultados semelhantes foram obtidos em corpos de prova de cerâmica vermelha com a incorporação de cinza do bagaço de cana de açúcar, sinterizados a mesma temperatura utilizada nesse estudo $\left(1050{ }^{\circ} \mathrm{C}\right.$; Borlini, Mendonça, Vieira \& Monteiro, 2006).

Segundo Gaspareto \& Teixeira (2017) que investigaram a incorporação de Resíduo de Construção Civil e Demolição (RCD) na massa cerâmica para a produção de tijolos maciços a presença de quartzo e compostos de cálcio, pode ter provocado o aumento do volume de microtrincas, consequentemente fragilizando material.

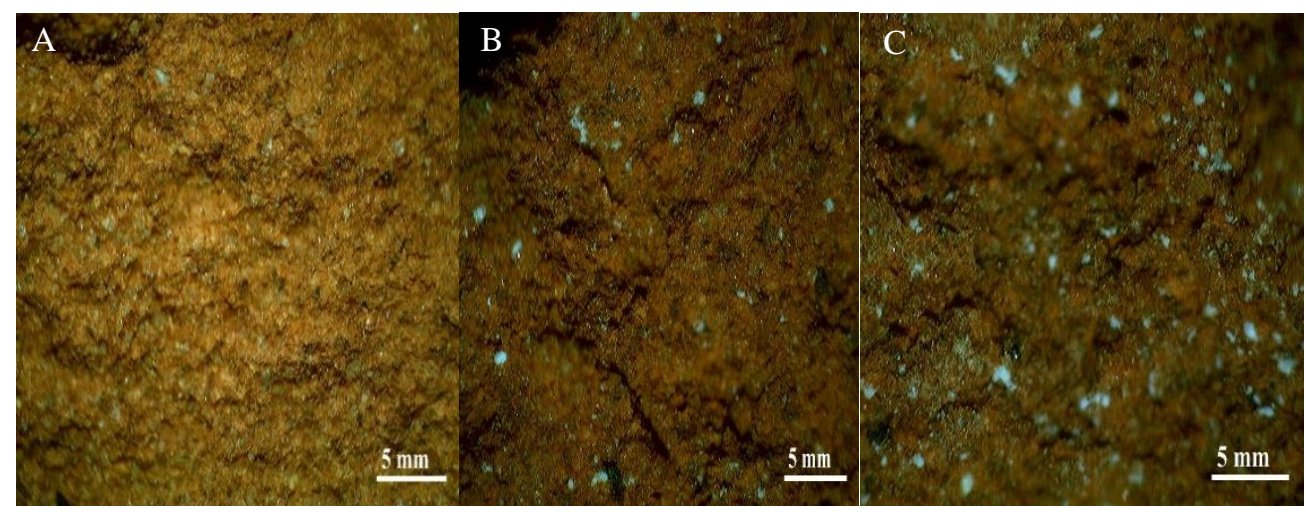

Figura 2. Micrografias obtidas da superfície de fratura de amostras de corpos de prova cerâmicos queimados a $1050{ }^{\circ} \mathrm{C}$, contendo somente argila (A), $5 \%$ (B) e $10 \%$ (C) de resíduo de gesso, com magnificação de 20x.

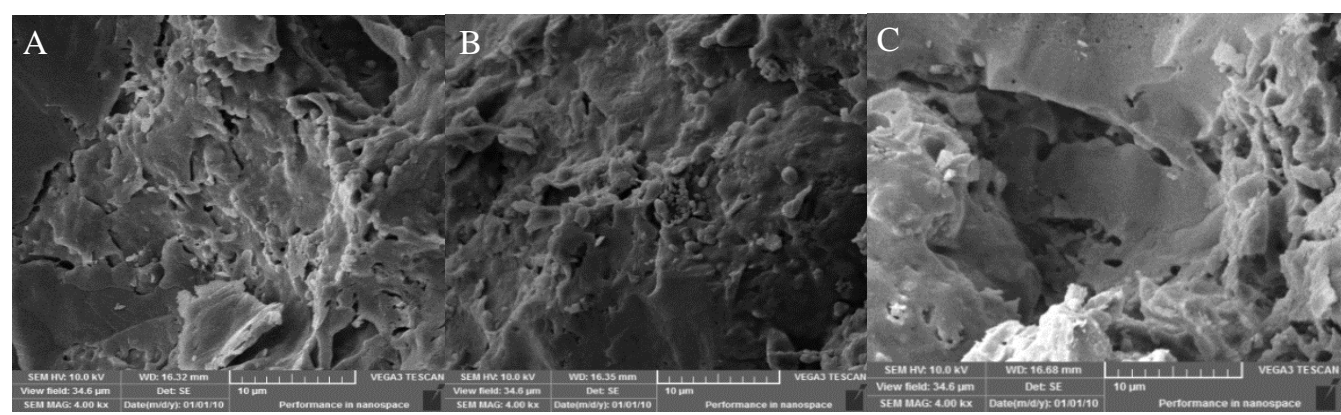

Figura 3. Micrografias obtidas em microscopia eletrônica de varredura da superfície de fratura de amostras de corpos de prova cerâmicos queimados a $1050^{\circ} \mathrm{C}$, contendo somente argila (A), $5 \%$ (B) e $10 \%$ (C) de resíduo de gesso.

\section{Conclusões}

A adição de resíduo de gesso na argila interferiu nas propriedades dos corpos de prova sinterizados aumentando a porosidade, a absorção de água e a perda ao fogo, consequentemente reduzindo a massa específica aparente. As micrografias mostraram a presença de aglomerados e de poros, que podem atuar como concentradores de tensões diminuindo a resistência à flexão. As propriedades foram mais 
influenciadas pela temperatura de sinterização e sua interação com o teor de resíduo de gesso. Melhores resultados foram obtidos com a adição de $5 \%$ de resíduo de gesso na massa cerâmica sinterizados a $800{ }^{\circ} \mathrm{C}$ apresentando propriedades com diferença não significativa à argila pura. A utilização de materiais residuais permite a redução dos impactos ambientais e a possibilidade de novas formulações para produtos de cerâmica vermelha.

\section{Agradecimentos}

A UNIVASF pela concessão da bolsa de iniciação científica (BIA). A CAPES, pela concessão da bolsa de pósdoutorado (PNPD). A Ecogesso pela doação da argila residual.

\section{Referências}

Araújo, S. M. S. (2004). O pólo gesseiro do Araripe: unidades geo-ambientais e impactos da mineração (Tese de doutorado). Universidade Estadual de Campina, Campinas, São Paulo, Brasil. Recuperado de http://repositorio.unicamp.br/jspui/handle/REPOSIP/286929

Associação Brasileira de Normas Técnicas. (2005). NBR 15270-1: Parte 1: Blocos cerâmicos para alvenaria de vedação, terminologia e requisitos. Rio de Janeiro: Associação Brasileira de Normas Técnicas. Recuperado de http://www.abntcatalogo.com.br/norma.aspx?ID=000580

Barbosa, A. A., Ferraz, A.V. \& Santos, G. A. (2014)._Caracterização química, mecânica e morfológica do gesso obtido do pólo do Araripe. Cerâmica, 60(356), 501-508. doi: 10.1590/S0366-69132014000400007

Borlini, M. C., Mendonça, J. L. C. C., Vieira, C. M. F. \& Monteiro, S. N. (2006). Influência da Temperatura de Sinterização nas Propriedades Físicas, Mecânicas e Microestruturais de Cerâmica Vermelha Incorporada com Cinza de Bagaço de Cana de Açúcar. Revista Matéria, 11(4), 435-443.

Cabral Jr., M. \& Azevedo, P. B. M. (2017). Potencial Técnico e Econômico do Aproveitamento de Resíduos da Indústria de Cerâmica Vermelha. Cerâmica Industrial, 22(3), 29-38. doi: 10.4322/cerind.2017.017

Carvalho, L. L., Gomes, P. R. B., Pinheiro, H. A., Lima, H. S. \& Silva, E. F. (2015). Gesso: uma perspectiva dos impactos ambientais, noções básicas sobre sua produção química e ponto estratégico de captação de renda na cidade de Grajaú-MA. Revista Educação Ambiental em Ação, 14(54). Recuperado de http://www.revistaea.org/artigo.php?idartigo $=2248$

Dutra, R. P. S., Silva, J. B., Varela, M. L., Nascimento, R. M., Gomes, U. U. \& Paskocimas, C. A. (2008). Avaliação da Potencialidade de Argilas do Rio Grande do Norte, Brasil. Parte-II: Propriedades Tecnológicas. Cerâmica Industrial, 13(1-2), 47-50.

Gaspareto, M. G. T. \& Teixeira, S. R. (2017). Utilização de Resíduo de Construção Civil e Demolição (RCD) como Material não Plástico para a Produção de Tijolos Cerâmicos. Cerâmica Industrial, Vol. 22 N. ${ }^{\circ}$ 2, pp. 40-46. doi: 10.4322/cerind.2017.014

Magalhães, J. M., Silva, J. E., Castro, F. P. \& Labrincha, J. A. (2004). Effect of experimental variables on the inertization of galvanic sludges in claybased ceramics. Journal of Hazardous Materials, 106(2-3), pp 139-147.

Menezes, R. R., Almeida, R. R. B., Santana, L. N. L., Ferreira, C. H. S. D., Neves, G. A. \& Ferreira, H. C. (2007). Utilização do Resíduo do Beneficiamento do Caulim na Produção de Blocos e Telhas Cerâmicos. Revista Matéria, 12(1), 226-236.

Moreno, M. M. T., Bartolomeu, D. \& Lima, R. H. C. (2009). Análise do comportamento de queima de argilas e formulações para revestimento cerâmico. Cerâmica, 55, 286-295. doi: 10.1590/S036669132009000300008

Morini, A. A., Oliveira, K. A., Pereira, F. R. \& Hotza, D. (2016). Avaliação da Potencialidade do Uso de Resíduos Industriais Através de Ferramenta de Seleção de Materiais para Projeto de Produtos Cerâmicos. Cerâmica Industrial, 21(2), 36-44. doi: 10.4322/cerind.2016.012

Pereira, I. D. S., Silva, I. A., Cartaxo, J. M., Menezes, R. R., Santana, L. N. L., Neves, G. A. \& Ferreira, H. C. (2014). Estudos de caracterização dos novos depósitos de argilas esmectíticas do município de Sossego, Paraíba, Brazil. Cerâmica, 60(354), 223-230. doi: 10.1590/S036669132014000200010
Pissato, E. \& Soares, L. (2009). Caracterização de Resíduos Finos de uma Mineração de Areia no Município de Guarulhos para Utilização em Cerâmica Vermelha. Cerâmica Industrial, 14(3), 41-46.

Queiroz, L. F. T., Monteiro, S. N. \& Vieira, C. M. F. (2010). Efeito da utilização de areia no processamento e nas propriedades de cerâmica vermelha. Cerâmica, 56, 279-284. doi: 10.1590/S036669132010000300011

Santos, S. P. (1992). Ciência e Tecnologia de Argilas (2a ed., pp. 91-408). São Paulo: Edgard Blücher.

Santos, R. C., Silva, T. R., Neves, G. A., Macedo, R. S., Menezes, R. R. e Santana, L. N. L. (2017). Interação entre características de argilas e parâmetros de processamento sobre propriedades tecnológicas de corpos cerâmicos. Cerâmica, 63, 361-368. doi: 10.1590/036669132017633672126

Santis, B. C., Sichieri, E. P., Rossignolo, J. A., Ferreira, G. \& Fiorelli, J. (2013). Caracterização de massas cerâmicas do estado de S. Paulo para produção de agregados leves para concreto. Cerâmica, 59(350), 198-205. doi: 10.1590/S0366-69132013000200002

Silva, J. B. (2008). Avaliação da Potencialidade dos Resíduos de Gesso de Revestimento Incorporados em Formulações de massas cerâmicas (Tese de doutorado). Universidade Federal do Rio Grande do Norte. Natal, Rio Grande do Norte, Brasil. Recuperado de https://repositorio.ufrn.br/jspui/handle/123456789/12717

Sousa, R. F. (2014). Obtenção e caracterização de um compósito a base de rejeito do gesso e chamote de telha para produção de filtros cerâmicos (Dissertação de mestrado). Universidade Federal do Rio Grande do Norte. Natal, Rio Grande do Norte, Brasil. Recuperado de https://repositorio.ufrn.br/jspui/handle/123456789/19292

Urbánek, J., Hamácek, J., Machácek, J., Kutzendörfer, J. \& Hubálková, J. (2017). Thermomechanical properties of mullitic materials. Processig and Apllication of Ceramics, 11(4), 322-328. doi: 10.2298/PAC $1704322 \mathrm{U}$

Vieira, A. W., Innocentinic, M. D. M., Mendes, E., Gomes, T., Demarch, A., Montedo, O. R. K. \& Angioletto, E. (2017). Comparison of Methods for Determining the Water Absorption of Glazed Porcelain Stoneware Ceramic Tiles. Materials Research, 20(Suppl. 2), 637-643. doi: 10.1590/1980-5373-MR-2017-0089

\section{Licença Creative Commons CC BY 4.0}

Este artigo foi publicado com acesso aberto para distribuíção sob os termos do Licença de Atribuição Creative Commons, que permite uso irrestrito, distribuição, e reprodução em qualquer meio, desde que o trabalho original seja devidamente citado. 